\title{
Connectivity Preserving State Agreement for Multiple Unicycles
}

\author{
Dimos V. Dimarogonas and Kostas J. Kyriakopoulos
}

\begin{abstract}
This paper presents a decentralized feedback control strategy that drives a system of multiple nonholonomic kinematic unicycles to agreement, maintaining at the same time the connectivity properties of the initially formed communication graph. The communication graph is created based on the initial relative distances between the team members. The proposed control law guarantees that if the communication graph is initially connected, then it remains connected throughout the closed loop system evolution. This is achieved via a control design that renders the set of edges of the initially formed communication graph positively invariant for the trajectories of the closed loop system. The proposed nonholonomic control law is discontinuous and time-invariant and tools from nonsmooth stability theory and matrix theory are used to check the stability of the overall system. The convergence properties are verified through computer simulations.
\end{abstract}

\section{INTRODUCTION}

Navigation of multi-agent systems is a topic that has recently attracted researchers from both the robotics and the control communities, due to the need for autonomous control of more than one mobile robotic agents in the same workspace. While most approaches in the past focused at centralized planning ([16]), specific real-world applications have lead researchers throughout the globe to turn their attention to decentralized concepts. One such important application is the field of micro robotics ([11]), where a team of a potentially large number of autonomous micro robots must cooperate in the sub micron level.

In this paper, the problem of rendezvous convergence for a system of multiple nonholonomic unicycles in terms of both position and orientation is considered. The rendezvous problem has been extensively approached recently, addressing the control design issue from several perspectives. Recent results include [21],[5],[25], [19],[15],[12]. In most cases, linear models of motion are taken into account, while the information exchange topology is considered both static and dynamic, as well as bidirectional or unidirectional. A recent review of the various approaches of the rendezvous problem for linear models of motion is [23].

In previous work we proposed an agreement strategy for multiple unicycles under static [6] and time-varying [7] communication topology. The assumption that the communication graph remained connected was a necessary condition for achieving agreement in [7]. An improved result is obtained in the current paper. Specifically, we propose a control strategy that guarantees that if the communication graph, which is formed based on the initial relative distances

\footnotetext{
The authors are with the Control Systems Lab, Department of Mechanical Engineering, National Technical University of Athens, 9 Heroon Polytechniou Street, Zografou 15780, Greece ddimar,kkyria@mail.ntua.gr
}

between the team members, is connected, then it remains connected throughout the closed loop system evolution. This is achieved via a control design that renders the set of edges of the initially formed communication graph positively invariant for the trajectories of the closed loop system. Connectivity preserving algorithms for multi-agent systems with linear models of motion have recently been dealt with in [13],[26],[20]. A comparison between the control design of the current paper and our previous work [6],[7] can be found in [8].

The proposed nonholonomic feedback law is discontinuous and time invariant, something expected, as nonholonomic systems do not satisfy Brocketts necessary smooth feedback stabilization condition [2]. These controllers have in general better convergence properties than time-varying ones. An experimental comparison between these two types of controllers that supports our preference to time-invariant strategies has appeared in [14], where the authors concluded that time-varying controllers were too slow and oscillatory for most practical cases. In contrast, time-invariant controllers achieved a significantly better performance. A timevarying control law for agreement of multiple unicycles was presented in [17].

The rest of the paper is organized as follows: section II describes the system and the problem that is treated in this paper. Assumptions regarding the communication topology between the agents are presented and modelled in terms of an undirected graph. Section III begins with some background on matrix and graph theory and nonsmooth analysis that is used in the sequel and proceeds with the introduction of the distributed nonsmooth time invariant feedback control strategy that drives the multi-agent team to a common configuration in the state space as well as the corresponding stability analysis. Computer simulation results are included in section IV while a summary of the results of this paper is provided in section $\mathrm{V}$.

\section{System And Problem Definition}

We consider a system of $N$ nonholonomic kinematic point agents operating in the same workspace $W \subset \mathbb{R}^{2}$. Let $q_{i}=\left[x_{i}, y_{i}\right]^{T} \in \mathbb{R}^{2}$ denote the position of agent $i$ and $q=$ $\left[q_{1}, \ldots, q_{N}\right]^{T}$ the stack vector of all agents positions. Each of the $N$ mobile agents has a specific orientation $\theta_{i}$ with respect to the global coordinate frame. The orientation vector of the agents is represented by $\theta=\left[\theta_{1} \ldots \theta_{N}\right]$. The configuration of each agent is represented by $p_{i}=\left[\begin{array}{cc}q_{i} & \theta_{i}\end{array}\right] \in \mathbb{R}^{2} \times(-\pi, \pi]$. Agent motion is described by the following nonholonomic 
kinematics:

$$
\begin{aligned}
& \dot{x}_{i}=u_{i} \cos \theta_{i} \\
& \dot{y}_{i}=u_{i} \sin \theta_{i} \quad, i \in \mathcal{N}=[1, \ldots, N] \\
& \quad \dot{\theta}_{i}=\omega_{i}
\end{aligned}
$$

where $u_{i}, \omega_{i}$ denote the translational and rotational velocity of agent $i$, respectively. These are considered as the control inputs of the multi-agent system.

The design objective is to construct feedback controllers that lead the multi-agent system to rendezvous, i.e. all agents should converge to a common point in the state space. Each agent is assigned with a subset $N_{i}$ of the rest of the team, called agent $i$ 's communication set, that includes the agents with which it can communicate in order to achieve the desired agreement objective. Inter-agent communication can be encoded in terms of a communication graph:

Definition 1: The communication graph $\mathcal{G}=\{V, E\}$ is an undirected graph that consists of a set of vertices $V=$ $\{1, \ldots, N\}$ indexed by the team members and (ii) a set of edges, $E=\left\{(i, j) \in V \times V \mid i \in N_{j}\right\}$ containing pairs of nodes that represent inter-agent communication specifications.

Furthermore, each agent has limited sensing capabilities which are bounded within a cyclic area of specific radius $d$ around the agent. This cyclic area is called the sensing zone, while the parameter $d$ the sensing radius of each agent.

In [7], we considered the case of varying interconnection topology between the members of the unicycle team, while the static case was dealt with in [6]. A necessary condition for the validity of the agreement control design of [7] was that the communication graph remained connected. In this paper, we modify that control design in order to guarantee that if the communication graph is initially connected, then it remains connected until rendezvous is reached. In particular, we show that the modified control design forces the agents that are initially located within the sensing radius of an agent to remain within this area for all time. In this way, no edges are lost and the initially connected communication graph remains connected for all time.

Hence in this paper, the set $N_{i}$ is now defined as the set that agent $i$ can sense when it is located at its initial position, $q_{i}(0)$ :

$$
N_{i}=\left\{j \in \mathcal{N}, j \neq i:\left\|q_{i}(0)-q_{j}(0)\right\|<d\right\}
$$

Each agent needs only knowledge of the state of agents that belong to its communication set. Moreover, the communication graph is undirected, in the sense that $i \in N_{j} \Leftrightarrow$ $j \in N_{i}, \forall i, j \in \mathcal{N}, i \neq j$. It is obvious that $(i, j) \in E$ iff $i \in N_{j} \Leftrightarrow j \in N_{i}$.

The control design is of the form

$$
\begin{aligned}
& u_{i}=u_{i}\left(p_{i}, p_{j}\right) \\
& \omega_{i}=\omega_{i}\left(p_{i}, p_{j}\right)
\end{aligned}, j \in N_{i}, i \in \mathcal{N}
$$

copying in this way with the limited communication capabilities of each agent. The problem treated in this paper can now be stated as follows: "under the preceding assumptions, derive a set of distributed control laws of the form (3) that drives the team of agents from any initial configuration to a common configuration in the state space".

\section{Control Design And Stability AnAlysis}

\section{A. Tools from Matrix Theory}

In this subsection we review some tools from graph theory and matrix analysis that we shall use in the stability analysis of the proposed control framework. The following analysis on graph theory can be found in [1], while the elements from matrix analysis in [10],[18].

For an undirected graph $\mathcal{G}=(V, E)$ with $n$ vertices we denote by $V$ its set of vertices and by $E$ its set of edges. If there is an edge connecting two vertices $i, j$, i.e. $(i, j) \in E$, then $i, j$ are called adjacent. A path of length $r$ from a vertex $i$ to a vertex $j$ is a sequence of $r+1$ distinct vertices starting with $i$ and ending with $j$ such that consecutive vertices are adjacent. If there is a path between any two vertices of the graph $\mathcal{G}$, then $\mathcal{G}$ is called connected (otherwise it is called disconnected).

The undirected graph $\mathcal{G}=(V, E)$ corresponding to a real symmetric $n \times n$ matrix $M$ is a graph with $n$ vertices indexed by $1, \ldots, n$ such that there is an edge between vertices $i, j \in$ $V$ if and only if $M_{i j} \neq 0$, i.e. $(i, j) \in E \Leftrightarrow M_{i j} \neq 0$.

A $n \times n$ real symmetric matrix with non-positive offdiagonal elements and zero row sums is called a symmetric Metzler matrix. It is shown in [18] that all the eigenvalues of a symmetric Metzler matrix are non-negative and zero is a trivial eigenvalue. The multiplicity of zero as an eigenvalue of a symmetric Metzler matrix is one (i.e. it is a simple eigenvalue) if and only if the corresponding undirected graph is connected. The trivial corresponding eigenvector is the vector of ones, $\overrightarrow{\mathbf{1}}$. This result has been used in the proof of the consensus algorithm for single integrator kinematic agents presented in [19]. Its usefulness in the present framework is verified in the sequel.

\section{B. Tools from Nonsmooth Analysis}

In this subsection, we review some elements from nonsmooth analysis and Lyapunov theory for nonsmooth systems that we use in the stability analysis of the next section.

For a differential equation with discontinuous right-hand side we have the following definition:

Definition 2: [9] In the case when the state-space is finite dimensional, the vector function $x($.$) is called a Filippov$ solution of $\dot{x}=f(x)$ if it is absolutely continuous and $\dot{x} \in$ $K[f](x)$ almost everywhere where

$$
K[f](x) \equiv \overline{c o}\left\{\lim _{x_{i} \rightarrow x} f\left(x_{i}\right) \mid x_{i} \notin N\right\}
$$

where $N$ is a set of measure zero.

Lyapunov stability theorems have been extended for nonsmooth systems in [24],[3]. The following chain rule provides a calculus for the time derivative of the energy function in the nonsmooth case:

Theorem 1: [24] Let $x$ be a Filippov solution to $\dot{x}=f(x)$ on an interval containing $t$ and $V: \mathbb{R}^{n} \rightarrow \mathbb{R}$ be a Lipschitz 
and regular function. Then $V(x(t))$ is absolutely continuous, $(d / d t) V(x(t))$ exists almost everywhere and

$$
\frac{d}{d t} V(x(t)) \in^{a . e .} \dot{\tilde{V}}(x):=\bigcap_{\xi \in \partial V(x(t))} \xi^{T} K[f](x(t))
$$

where "a.e." stands for "almost everywhere".

In this theorem, $\partial V$ is Clarke's generalized gradient. The definition of the generalized gradient and of the regularity of a function can be found in [4]. In the case we encounter in this paper, the candidate Lyapunov function $V$ we use is smooth and hence regular, while its generalized gradient is a singleton which is equal to its usual gradient everywhere in the state space: $\partial V(x)=\{\nabla V(x)\} \forall x \in \mathbb{R}^{n}$.

We shall use the following nonsmooth version of LaSalle's invariance principle to prove the convergence of the prescribed system:

Theorem 2: [24] Let $\Omega$ be a compact set such that every Filippov solution to the autonomous system $\dot{x}=$ $f(x), x(0)=x\left(t_{0}\right)$ starting in $\Omega$ is unique and remains in $\Omega$ for all $t \geq t_{0}$. Let $V: \Omega \rightarrow \mathbb{R}$ be a time independent regular function such that $v \leq 0 \forall v \in \dot{\widetilde{V}}$ (if $\dot{\vec{V}}$ is the empty set then this is trivially satisfied). Define $S=\{x \in \Omega \mid 0 \in \dot{\widetilde{V}}\}$. Then every trajectory in $\Omega$ converges to the largest invariant set, $M$, in the closure of $S$.

\section{Control Law and Stability Analysis}

Existence of an edge between agents $i$ and $j$ is now reflected in the following attractive potential:

$$
\delta_{i j}\left(\beta_{i j}\right)=\left\{\begin{array}{l}
\frac{a_{3}}{d^{2}-\beta_{i j}}, \mu<\beta_{i j}<d^{2} \\
a_{4} \beta_{i j}, 0 \leq \beta_{i j} \leq \mu
\end{array}\right.
$$

where $\beta_{i j} \triangleq\left\|q_{i}-q_{j}\right\|^{2}$ the squared norm of the Euclidean distance of agents $i, j$. The parameters of $\delta_{i j}$ are chosen so that it is continuously differentiable for all $\beta_{i j}<d^{2}$. It is easily derived that this is fulfilled provided that

$$
d^{2}=2 \mu, a_{3}=a_{4} \mu^{2}
$$

Figure 1 shows a plot of the function $\delta_{i j}$ with respect to $\beta_{i j}$ for $d^{2}=0.00067$.

The total attractive potential of agent $i$ is given by

$$
\delta_{i}=\sum_{j \in N_{i}} \delta_{i j}\left(\beta_{i j}\right)
$$

The gradient with respect to $q$ and the partial derivative of $\delta_{i j}$ with respect to $q_{i}$ are computed by

$$
\nabla \delta_{i j}=2 p_{i j} D_{i j} q, \frac{\partial \delta_{i j}}{\partial q_{i}}=2 p_{i j}\left(D_{i j}\right)_{i} q
$$

where

$$
p_{i j} \triangleq \frac{\partial \delta_{i j}}{\partial \beta_{i j}}
$$

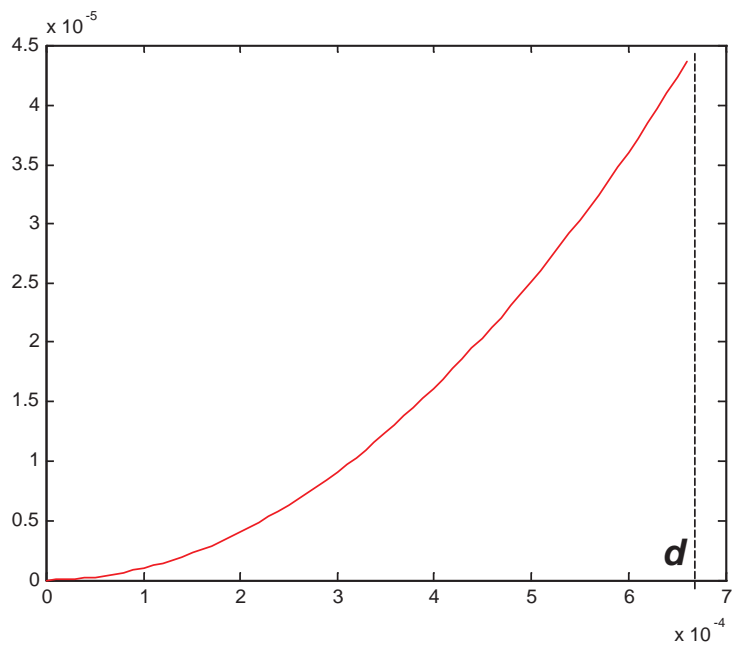

Fig. 1. The function $\delta_{i j} d^{2}=0.00067$.

and the matrices $D_{i j},\left(D_{i j}\right)_{i}$, for $i<j$, can be shown to be given by

$$
\begin{aligned}
& D_{i j}= \\
& {\left[\begin{array}{ccccc}
O_{1 \times(i-1)} & 1 & O_{1 \times(j-i-1)} & -1 & O_{1 \times(N-j)} \\
& & O_{(j-i-1) \times N} & & \\
O_{1 \times(i-1)} & -1 & O_{1 \times(j-i-1)} & 1 & O_{1 \times(N-j)} \\
& & O_{(N-j) \times N} & &
\end{array}\right] \otimes I_{2}}
\end{aligned}
$$

and

$$
\begin{aligned}
& \left(D_{i j}\right)_{i}= \\
& {\left[\begin{array}{lllll}
O_{1 \times(i-1)} & 1 & O_{1 \times(j-i-1)} & -1 & O_{1 \times(N-j)}
\end{array}\right] \otimes I_{2}}
\end{aligned}
$$

The definition of the matrices $D_{i j},\left(D_{i j}\right)_{i}$, for $i>j$ is straightforward.

Define now

$$
\delta=\sum_{i} \delta_{i}=\sum_{i} \sum_{j \in N_{i}} \delta_{i j}\left(\beta_{i j}\right)
$$

Taking the gradient of $\delta$ with respect to $q$ we get

$$
\begin{aligned}
& \nabla \delta=\sum_{i} \sum_{j \in N_{i}} \nabla \delta_{i j}=2\left(\sum_{i} \sum_{j \in N_{i}} p_{i j} D_{i j}\right) q= \\
& =4\left(P \otimes I_{2}\right) q
\end{aligned}
$$

where the $N \times N$ matrix $P$ is easily shown to be given by

$$
P_{i j}=\left\{\begin{array}{l}
\sum_{j \in N_{i}} p_{i j}, j=i \\
-p_{i j}, j \in N_{i}, i \neq j \\
0, j \notin N_{i}
\end{array}\right.
$$

The previous result was derived based on the following symmetry property:

$$
p_{i j}=p_{j i}, \forall j \in N_{i}
$$

We also have

$$
\frac{\partial \delta_{i}}{\partial q_{i}}=\sum_{j \in N_{i}} \frac{\partial \delta_{i j}}{\partial q_{i}}
$$


and an easy calculation yields

$$
\left[\begin{array}{c}
\frac{\partial \delta_{1}}{\partial q_{1}} \\
\vdots \\
\frac{\partial \delta_{N}}{\partial q_{N}}
\end{array}\right]=\left[\begin{array}{c}
\sum_{j \in N_{1}} \frac{\partial \delta_{1 j}}{\partial q_{1}} \\
\vdots \\
\sum_{j \in N_{N}} \frac{\partial \delta_{N j}}{\partial q_{N}}
\end{array}\right]=-2\left(P \otimes I_{2}\right) q
$$

In the analysis that follows, we use the decoupling of the stack vector $q=[x, y]^{T}$ into the coefficients that correspond to the $x, y$ directions of the agents respectively. Furthermore, the notation $(a)_{i}$ for a vector $a$, denotes its $i$-th element.

We propose the following discontinuous time-invariant feedback control strategy for each agent $i$ :

$$
\begin{gathered}
u_{i}=-\operatorname{sgn}\left\{\delta_{x i} \cos \theta_{i}+\delta_{y i} \sin \theta_{i}\right\} \cdot\left(\delta_{x i}^{2}+\delta_{y i}^{2}\right)^{1 / 2} \\
\omega_{i}=-\left(\theta_{i}-\theta_{n h_{i}}\right)
\end{gathered}
$$

where

$$
\begin{aligned}
& \delta_{x i}=\frac{\partial \delta_{i}}{\partial x_{i}}=(2 P x)_{i}=2 \sum_{j \in N_{i}} p_{i j}\left(x_{i}-x_{j}\right), \\
& \delta_{y i}=\frac{\partial \delta_{i}}{\partial y_{i}}=(2 P y)_{i}=2 \sum_{j \in N_{i}} p_{i j}\left(y_{i}-y_{j}\right)
\end{aligned}
$$

and

$$
\theta_{n h_{i}}=\arctan 2\left(\delta_{y i}, \delta_{x i}\right)
$$

We use the continuously differentiable positive definite function $W=\delta$ as a candidate Lyapunov function.

Since the proposed control law is discontinuous we use the concept of Theorem 1 for the time derivative of the candidate Lyapunov function. Since $W$ is smooth we have $\partial W=\{\nabla W\}=\{\nabla \delta\}$, so that

$$
\begin{aligned}
& \dot{\widetilde{W}}=\left\{\sum_{i}\left(\nabla \delta_{i}\right)^{T}\right\} \cdot K\left[\begin{array}{c}
u_{1} \cos \theta_{1} \\
u_{1} \sin \theta_{1} \\
\vdots \\
u_{N} \cos \theta_{N} \\
u_{N} \sin \theta_{N}
\end{array}\right] \subset \\
& 4 q^{T}\left(P \otimes I_{2}\right)\left[\begin{array}{c}
K\left[u_{1}\right] \cos \theta_{1} \\
K\left[u_{1}\right] \sin \theta_{1} \\
\vdots \\
K\left[u_{N}\right] \cos \theta_{N} \\
K\left[u_{N}\right] \sin \theta_{N}
\end{array}\right] \subset \\
& \subset 4(P x)^{T}\left[\begin{array}{c}
K\left[u_{1}\right] \cos \theta_{1} \\
\vdots \\
K\left[u_{N}\right] \cos \theta_{N}
\end{array}\right]+ \\
& +4(P y)^{T}\left[\begin{array}{c}
K\left[u_{1}\right] \sin \theta_{1} \\
\vdots \\
K\left[u_{N}\right] \sin \theta_{N}
\end{array}\right] \subset \\
& \subset \sum_{i}\left\{4 K\left[u_{i}\right]\left((P x)_{i} \cos \theta_{i}+(P y)_{i} \sin \theta_{i}\right)\right\}
\end{aligned}
$$

where we used Theorem 1.3 in [22] to calculate the inclusions of the Filippov set in the previous analysis. Since $K[\operatorname{sgn}(x)] x=\{|x|\}([22]$, Theorem 1.7), the choice of control laws (4),(5) results in

$$
\dot{\widetilde{W}}=-\sum_{i}\left\{8\left|\delta_{x i} \cos \theta_{i}+\delta_{y i} \sin \theta_{i}\right|\left(\delta_{x i}^{2}+\delta_{y i}^{2}\right)^{1 / 2}\right\} \leq 0
$$

so that the generalized derivative of $W$ reduces to a singleton. The last equation implies that $W$ is non-increasing across the trajectories of the closed loop system, i.e. $W(q(t)) \leq$ $W(q(0))$ for all $t \geq 0$.

The first result of this paper involves the invariance of the initially formed communication graph:

Lemma 3: Consider the system of multiple kinematic unicycles (1) driven by the control law (4),(5). The set $\mathcal{J}(q)=\left\{q \mid\left\|q_{i}-q_{j}\right\| \leq d, \forall(i, j) \in E\right\}$ is invariant for the trajectories of the closed loop system.

Proof: For every initial condition $q(0) \in \mathcal{J}(q)$, we have $W(q(t)) \leq W(q(0))<\infty$ for all $t \geq 0$. Since $W \rightarrow \infty$ when $\left\|q_{i}-q_{j}\right\| \rightarrow d$ for at least one pair $(i, j) \in E$, we conclude that $q(t) \in \mathcal{J}(q)$, for all $t \geq 0$. $\diamond$

This control law guarantees that the initial set of edges, created under (2), remains invariant during the evolution of the closed loop system. Hence no edges are lost and no new ones are created, even when an agent, not initially located in the sensing zone of another, enters in this area at some time $t>0$. The sets $N_{i}$ initially formed remain constant as the system evolves.

We now state the main result of this paper:

Theorem 4: Assume that the communication graph formed under the initial condition ruling (2) is connected. Then the discontinuous time-invariant feedback control strategy (4),(5) drives the agents to a common configuration in the state space.

Proof: The level sets of $W$ are compact and invariant with respect to the relative positions of adjacent agents. Specifically, the set $\Omega_{c}=\{q: W(q) \leq c\}$ for $c>0$ is closed by the continuity of $W$. For all $(i, j) \in E$ we have $W \leq c \Rightarrow \delta_{i j} \leq c \Rightarrow\left\|q_{i}-q_{j}\right\| \leq \sqrt{\delta_{i j}^{-1}(c)}$. Connectivity of the initially formed communication graph guarantees that every pair $i, j$ of agents satisfies $\left\|q_{i}-q_{j}\right\| \leq(N-1) \delta_{\max }^{-1}$, where $\delta_{\max }^{-1} \triangleq \max _{(i, j) \in E} \sqrt{\delta_{i j}^{-1}(c)}$. Therefore, the set $\Omega_{c}$ is also bounded with respect to the relative positions of agents in the group. Hence for all initial conditions satisfying $\left\|q_{i}(0)-q_{j}(0)\right\|<d, \forall(i, j) \in E$ we can apply (the nonsmooth version of) LaSalle's invariance principle.

By the nonsmooth version of LaSalle's invariance principle (Theorem 2), the trajectories of the system converge to the largest invariant set contained in the set

$$
S=\left\{\begin{array}{r}
\left(\delta_{x i}=\delta_{y i}=0\right) \vee\left(\delta_{x i} \cos \theta_{i}+\delta_{y i} \sin \theta_{i}=0\right), \\
\forall i \in N
\end{array}\right\}
$$

However, for each $i \in \mathcal{N}$, we have $\left|\omega_{i}\right|=\frac{\pi}{2}$ whenever $\delta_{x i} \cos \theta_{i}+\delta_{y i} \sin \theta_{i}=0$, due to the proposed angular velocity control law. In particular, this choice of angular velocity renders the surface $\delta_{x i} \cos \theta_{i}+\delta_{y i} \sin \theta_{i}=0$ noninvariant for agent $i$, whenever $i$ is not located at the desired equilibrium, namely when $\delta_{x i}=\delta_{y i}=0$. Hence the largest invariant set $S_{o}$ contained in $S$ is

$$
S \supset S_{o}=\left\{\delta_{x i}=\delta_{y i}=0, \forall i \in \mathcal{N}\right\}
$$

In addition $\left(\delta_{x i}=\delta_{y i}=0\right) \forall i$ guarantees that the agents converge to a common configuration. This is easily derived 
by the fact that

$$
\left(\delta_{x i}=\delta_{y i}=0\right) \forall i \Rightarrow\left(P \otimes I_{2}\right) q=0 \Rightarrow P x=P y=0
$$

where $x, y$ the stack vectors of $q$ in the $x, y$ directions. Within $S_{o}$, the closed loop equations for the orientations have the form $\dot{\theta}_{i}=-\theta_{i}$ for all $i$, and hence the orientations of all agents tend to zero. The symmetric matrix $P$ has zero row sums and non-positive off-diagonal elements. Using the same arguments and terminology as in [19], the matrix $P$ is a Metzler matrix. As mentioned in Section IIIA, the eigenvalues of $P$ are nonnegative and zero is the smallest eigenvalue. Following [19], we deduce that since the initially formed communication graph is connected, zero is a simple eigenvalue of $P$ with trivial corresponding eigenvector the vector of ones, $\overrightarrow{\mathbf{1}}$. Hence equations $P x=P y=0$ guarantee that both $x, y$ are eigenvectors of $P$ belonging to $\operatorname{span}\{\overrightarrow{\mathbf{1}}\}$. Hence all $q_{i}$ tend to the same value, implying that all agents converge to a common configuration at steady state. $\diamond$

Remark: It must be stressed out that the proposed feedback control strategy (4),(5) is purely decentralized, since each agent requires information only of the states of agents within its initially formed communication set $N_{i}$. This control law guarantees that the initial set of edges, created under (2), remains invariant during the evolution of the closed loop system. Hence no edges are lost and no new ones are created, even when an agent, not initially located in the sensing zone of another, enters in this area at some time $t>0$. The sets $N_{i}$ initially formed remain constant as the system evolves.

\section{Simulations}

To support the results of the previous paragraphs we provide a computer simulation of the proposed control framework (4),(5).

The simulation involves a team of six unicycles with controller parameters $a_{4}=1, d^{2}=7.5 \cdot 10^{-3}$. The initial conditions for the six unicycles are given by

$$
\begin{aligned}
& p_{1}=\left[\begin{array}{lll}
-0.05 & 0.05 & \pi / 3
\end{array}\right]^{T}, \\
& p_{2}=\left[\begin{array}{lll}
-0.1 & -0.05 & \pi / 3
\end{array}\right]^{T}, \\
& p_{3}=\left[\begin{array}{lll}
-0.08 & 0.06 & -\pi / 4
\end{array}\right]^{T}, \\
& p_{4}=\left[\begin{array}{lll}
-0.1 & 0 & -3 \pi / 8
\end{array}\right]^{T}, \\
& p_{5}=\left[\begin{array}{lll}
0 & -0.05 & 3 \pi / 8
\end{array}\right]^{T}, \\
& p_{6}=\left[\begin{array}{lll}
0.1 & 0.12 & -7 \pi / 8
\end{array}\right]^{T}
\end{aligned}
$$

Please note that although the initially formed communication graph is connected, connectivity is lost if we apply the control design of [7]. In this case however, agreement is successfully achieved due to the connectivity invariance of the initially formed communication graph, as imposed by the control design of this paper. Screenshots I-V of Figure 2 show the evolution in time of the six unicycles under the proposed control strategy (4),(5). In the first screenshot, the agents are located at their initial positions. The pairs of agents that form edges in the initially formed communication graph are connected with a black line. Agents reach a rendezvous point in the last screenshot V. A plot of the cost functions $\delta_{i}$ for each agent is shown in Figure 3.
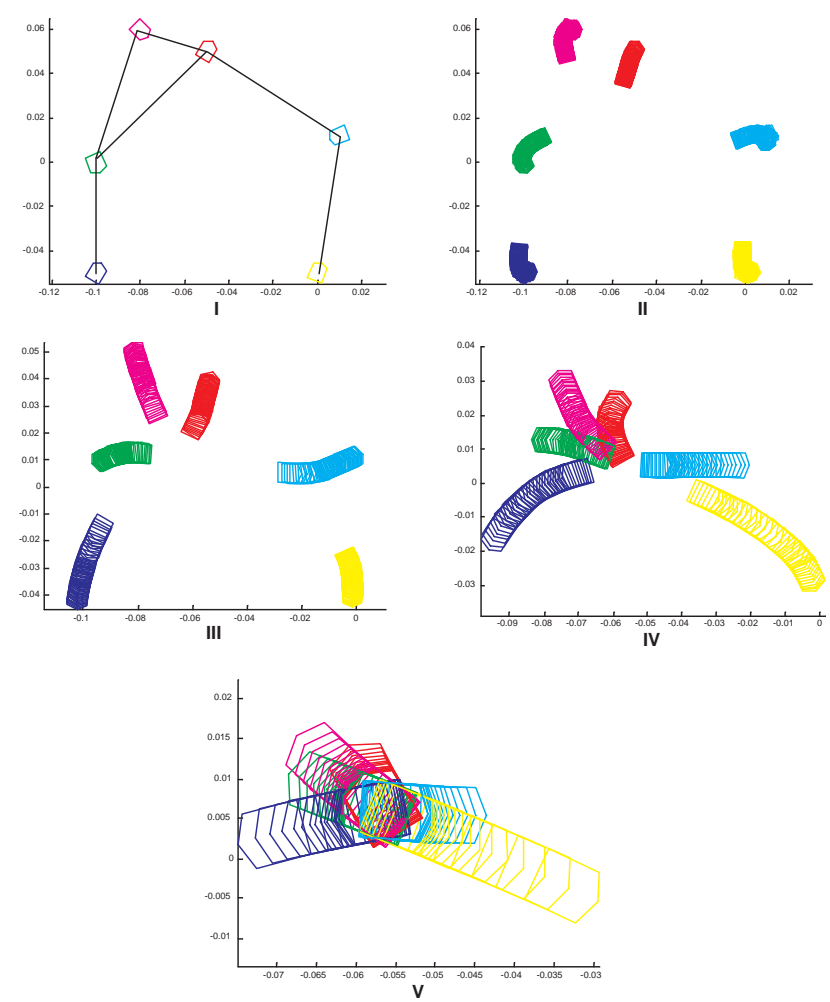

Fig. 2. Convergence to a common configuration for the six unicycles.

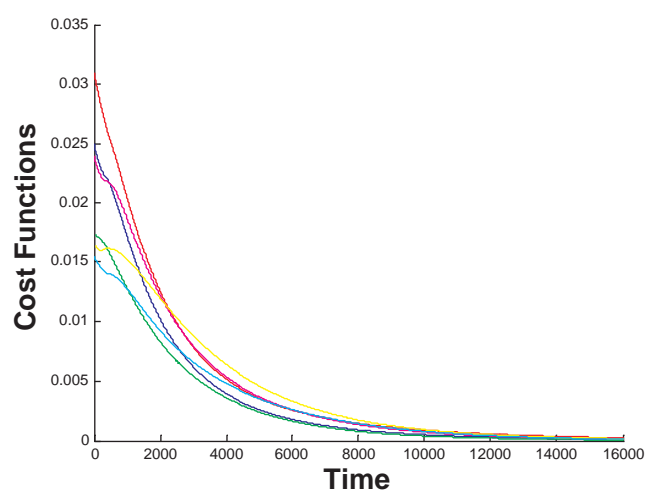

Fig. 3. Cost Functions $\delta_{i}$ for the six nonholonomic agents.

\section{Conclusions}

We presented a decentralized feedback control strategy that drives a system of multiple nonholonomic kinematic unicycles to agreement and maintains at the same time the connectivity properties of the initially formed communication graph. The communication graph is created based on the initial relative positions between the team members. The proposed control law guarantees that if the communication graph is initially connected, then it remains connected throughout the closed loop system evolution. This is achieved via a control design that renders the set of edges of the initially formed communication graph positively invariant for the trajectories of the closed loop system. The proposed nonholonomic 
control law is discontinuous and time-invariant and tools from nonsmooth stability theory and matrix theory are used to check the stability of the overall system. The convergence properties are verified through computer simulations.

Current research aims at taking into account the case of directed graphs. In addition, although the proposed control law is edge preserving, it does not take into account new agents entering the sensing zone of an agent, i.e. it does not allow for new edges to be created in the communication graph. Although new edge addition does not alter the convergence properties of the proposed scheme, it would improve the convergence rate and hence is a worthwhile research direction.

\section{ACKNOWLEDGEMENTS}

The authors would like to acknowledge the contribution of the European Commission through contract I-SWARM (IST2004-507006).

\section{REFERENCES}

[1] B. Bollobás. Modern Graph Theory. Springer Graduate Texts in Mathematics \# 184, 1998.

[2] R. W. Brockett. Control theory and singular riemannian geometry. In New Directions in Applied Mathematics, pages 11-27. Springer, 1981.

[3] F. Ceragioli. Discontinuous Ordinary Differential Equations and Stabilization. PhD thesis, Dept. of Mathematics, Universita di Firenze, 1999.

[4] F. Clarke. Optimization and Nonsmooth Analysis. Addison - Wesley, 1983.

[5] J. Cortes, S. Martinez, and F. Bullo. Robust rendezvous for mobile autonomous agents via proximity graphs in arbitrary dimensions. IEEE Transactions on Automatic Control, 51(8):1289-1298, 2006.

[6] D.V. Dimarogonas and K.J. Kyriakopoulos. On the state agreement problem for multiple unicycles. 2006 American Control Conference, pages 2016-2021.

[7] D.V. Dimarogonas and K.J. Kyriakopoulos. On the state agreement problem for multiple unicycles with varying communication links. 45th IEEE Conf. Decision and Control, pages 4283-4288, 2006.

[8] D.V. Dimarogonas and K.J. Kyriakopoulos. On the rendezvous problem for multiple nonholonomic agents. IEEE Transactions on Automatic Control, 2007. to appear.

[9] A. Filippov. Differential equations with discontinuous right-hand sides. Kluwer Academic Publishers, 1988.

[10] R. A. Horn and C. R. Johnson. Matrix Analysis. Cambridge University Press, 1996.

[11] Project ISWARM. http://microrobotics.ira.uka.de/.

[12] A. Jadbabaie, J. Lin, and A.S. Morse. Coordination of groups of mobile autonomous agents using nearest neighbor rules. IEEE Transactions on Automatic Control, 48(6):988-1001, 2003.

[13] M. Ji and M. Egerstedt. Connectedness preserving distibuted coordination control over dynamic graphs. 2005 American Control Conference, pages $93-98$.

[14] B. Kim and P. Tsiotras. Controllers for unicycle-type wheeled robots: Theoretical results and experimental validation. IEEE Transactions on Robotics and Automation, 18(3):294-307, 2002.

[15] G. Lafferriere, A. Williams, J. Caughman, and J.J.P. Veerman. Decentralized control of vehicle formations. Systems and Control Letters, 54(9):899-910, 2005.

[16] J. C. Latombe. Robot Motion Planning. Kluwer Academic Publishers, 1991.

[17] Z. Lin, B. Francis, and M. Maggiore. On the state agreement problem for multiple nonlinear dynamical systems. 16th IFAC World Congress, 2005.

[18] D.G. Luenberger. Introduction to Dynamic Systems: Theory, Models and Applications. John Wiley \& Sons, 1979.

[19] L. Moreau. Stability of continuous-time distributed consensus algorithms. 43rd IEEE Conf. Decision and Control, pages 3998-4003, 2004.
[20] G. Notarstefano, K. Savla, F. Bullo, and A. Jadbabaie. Maintaining limited-range connectivity among second-order agents. 2006 American Control Conference, pages 2124-2129.

[21] R. Olfati-Saber and R.M. Murray. Consensus problems in networks of agents with switching topology and time-delays. IEEE Transactions on Automatic Control, 49(9):1520-1533, 2004.

[22] B. Paden and S. S. Sastry. A calculus for computing Filippov's differential inclusion with application to the variable structure control of robot manipulators. IEEE Trans. on Circuits and Systems, 34(1):7382, 1987.

[23] W. Ren, R. W. Beard, and E. M. Atkins. A survey of consensus problems in multi-agent coordination. 2005 American Control Conference, pages $1859-1864$.

[24] D. Shevitz and B. Paden. Lyapunov stability theory of nonsmooth systems. IEEE Trans. on Automatic Control, 49(9):1910-1914, 1994.

[25] H.G. Tanner, A. Jadbabaie, and G.J. Pappas. Flocking in fixed and switching networks. IEEE Transactions on Automatic Control, submitted for publication, 2005.

[26] M.M. Zavlanos and G.J. Pappas. Controlling connectivity of dynamic graphs. 44th IEEE Conf. Decision and Control, pages 6388-6393, 2005. 